\title{
Norepinephrine influences the circadian clock in human dermal fibroblasts from study participants with a diagnosis of attention-deficit hyperactivity disorder
}

\author{
Denise Palm ${ }^{1} \cdot$ Adriana Uzoni $^{1} \cdot$ Frederick Simon $^{1} \cdot$ Oliver Tucha $^{1} \cdot$ Johannes Thome $^{1} \cdot$ Frank Faltraco $^{1}$
}

Received: 12 January 2021 / Accepted: 27 June 2021 / Published online: 18 July 2021

(c) The Author(s) 2021

\begin{abstract}
Attention-deficit hyperactivity disorder (ADHD) is characterized by changes to the circadian process. Many medications used to treat the condition, influence norepinephrine levels. Several studies have, in addition, reported that norepinephrine itself has an effect on circadian function. The aim of this study was to investigate the circadian gene expression in primary human-derived dermal fibroblast cultures (HDF) after norepinephrine exposure. We analyzed circadian preference, behavioral circadian and sleep parameters as well as the circadian gene expression in a cohort of healthy controls and participants with an ADHD diagnosis. Circadian preference was evaluated with German Morningness-Eveningness Questionnaire (D-MEQ) and rhythms of sleep/wake behavior were assessed via actigraphy. After ex vivo exposure to different norepinephrine concentrations in HDF cultures, the rhythmicity of circadian gene expression was analyzed via qRT-PCR. The exposure of $1 \mu \mathrm{M}$ norepinephrine to confluent cultures of human dermal fibroblasts from participants with a diagnosis of ADHD, was shown to dampen Perl rhythmicity. The expression of Bmall, Perl and Per3 in control subjects was also influenced by incubation with $1 \mu \mathrm{M}$ norepinephrine. Cultures from the ADHD group revealed no statistically significant overall differences in circadian gene expression, between cultures with and without norepinephrine incubation. Per3 expression showed a significant ZT $\times$ group interaction via mixed ANOVA. Per 3 expression at ZT4 was significant higher in the group of control samples incubated with $1 \mu \mathrm{M}$ norepinephrine, compared to the control group without norepinephrine. This effect was also shown in the control samples incubated with $1 \mu \mathrm{M}$ norepinephrine and cultures from subjects with ADHD without norepinephrine incubation. Per3 expression differed between the healthy control group and the ADHD group without norepinephrine incubation at ZT28. The results of the present study illustrate that norepinephrine impacts on circadian function. In both groups, control group and cultures taken from subjects with ADHD, the expression of the periodic genes (Perl-3) was significantly influenced by incubation with norepinephrine.
\end{abstract}

Keywords Norepinephrine $\cdot$ Human dermal fibroblasts $\cdot$ Circadian rhythm $\cdot$ Attention-deficit hyperactivity disorder

\section{Introduction}

The regulation of the norepinephrine (NE) homeostasis as well as the NE re-uptake into presynaptic nerve terminals among other substances is mediated by the NE transporter (NET) (Xu et al. 2000). Norepinephrine, like dopamine and epinephrine, is a catecholamine neurotransmitter derived

Frank Faltraco

frank.faltraco@med.uni-rostock.de

1 Department of Psychiatry and Psychotherapy, University Medical Centre Rostock, Rostock, Gehlsheimer Str. 20, 18147 Rostock, Germany from the common precursor amino acid tyrosine. Tyrosine is converted to L-dihydroxyphenylalanine (Dopa), after which Dopa is then converted to dopamine and transported to synaptic vesicles. Dopamine is converted to epinephrine, which is released into the cytosol for conversion to NE and after pre-synaptic release interacting with adrenoceptors (Xing et al. 2016). NE binds to the $\alpha 2$-adrenoceptors ( $\alpha 2 \mathrm{~A}$, $\alpha 2 \mathrm{~B}$ and the $\alpha 2 \mathrm{C}$ ) with highest affinity. In the skin, NE also binds to $\beta 2$-adrenoceptor expressed on the surface of keratinocytes, dermal fibroblasts and melanocytes (Gillbro et al. 2004; MacDonald et al. 1997; Steinkraus et al. 1996).

$\mathrm{NE}$ has been demonstrated to influence mammalian circadian rhythmicity. The human inner clock is generated by a master central clock in the suprachiasmatic nuclei (SCN) of 
the hypothalamus. Circadian rhythm is regulated by the main circadian genes (circadian locomotor output cycle kaput gene-Clock, brain and muscle Arnt-like 1 gene-Bmall, periodic genes-Per1/2/3 and cryptochrome genes-Cryl/2) that exhibit an auto-regulatory negative feedback loop, processed by signals like cyclic hormone production, as well as influenced by environmental changes such as light exposure (Czeisler et al. 1999; Moore 1997; Reppert and Weaver 2002). NE is partly responsible for the metabolic activity of the pineal gland and the nocturnal melatonin stimulation (Simonneaux and Ribelayga 2003; Terbeck et al. 2016).

Transient expression of Perl in astrocytes is induced in vitro by $\beta 2$-adrenoceptor activation through NE (Morioka et al. 2010). Studies have also demonstrated that NE can reactivate the circadian rhythm of adult rat cardiomyocytes, as well as regulate the physiological expression of mPerl, $m P e r 2$ and $m B m a l l$ in mice livers, and the rhythmic oscillation of $g$ Bmall, gClock, $g$ Cryl and $g$ Cry 2 in chicken pineal glands (Durgan et al. 2005; Li and Cassone 2015; Terazono et al. 2003). Andrade-Silva et al., a group that studied the circadian gene expression in rat pineal gland cultures in respect to NE synchronization, concluded that NE synchronization mimics its natural release in pineal glands (Andrade-Silva et al. 2014).

Several studies based on cell culture models have demonstrated that alterations in circadian gene expression as well as circadian rhythm disturbances are associated with neuropsychiatric disorders (Cronin et al. 2017; Johansson et al. 2016; Lippert et al. 2014; Mansour et al. 2017; McCarthy et al. 2013a, b; Yang et al. 2009). Alterations in Per2 and Cryl expression between individuals with a diagnosis of ADHD with no medication compared to medicated and healthy controls using human dermal fibroblasts as cellular model were reported (Coogan et al. 2019).

Human-derived fibroblasts provide an advantageous model to study the influence of drugs on circadian gene expression. The synchronization of the circadian system of fibroblasts can be synchronized by different substances, e.g. dexamethasone or NE. The effects of synchronizers are different on circadian gene expression (Faltraco et al. 2020).

NE also affects neurodegenerative and other psychiatric disorders (Marien et al. 2004). It is involved in the modulation of attention, arousal and cognition. Disturbances in the NE network are hypothesized to be associated with the pathophysiology of attention-deficit hyperactivity disorder (ADHD) (Sharma and Couture 2014). Abnormal regulation in NE neurotransmission as well as polymorphisms in the NET gene is reported to provide a link between NE and ADHD. In this respect, NE has been suggested to be associated with attention, alertness and executive function. In ADHD, inattentiveness and disturbances in executive functions are characteristic symptoms (Beane and Marrocco 2004; Biederman and Spencer 1999).
This leads to anticipation of an association between NE regulation and circadian gene expression as well as ADHD; and we hypothesized, that NE exposure influences the expression of circadian genes and thus may influence sleep quality and ADHD symptoms. Goal of this study is to investigate the effects of NE on circadian rhythmicity using the fibroblast model.

\section{Materials and methods}

\section{Participant selection criteria}

Ethical approval for the conduct of the study, including obtaining human dermal biopsy samples, was given by the ethical review committee of Rostock University (Registration-number: A2013-159) and written consent was obtained from each study participant. The study was conducted according to the ethical guidelines of the Declaration of Helsinki.

Subjects with ADHD and healthy controls participating in the study were recruited via the Department of Psychiatry and Psychotherapy, University Medical Centre Rostock. All subjects with ADHD were diagnosed by experienced psychiatrists in advance. The control group was recruited of acquaintances of people involved in the study.

Human dermal fibroblasts (HDF) were obtained from skin biopsies from dorsal forearm of subjects with ADHD and control volunteers. Only adult individuals, able to give informed consent, were included. Controls without a history of childhood or adult ADHD were matched for sex and age. Patients with debilitating psychiatric symptoms were excluded. Shift workers were also excluded. Screening for ADHD symptoms was done using the WURS-k (Wender Utah Rating Scale) as well as assessment of symptoms according to DSM-IV and ICD-10 criteria. Additionally, the following psychometric tests were used to confirm ADHD diagnosis: SKIDI and II (structured clinical interview), DIVA 2.0 (structured diagnostic interview), CAARS (Conners' Adult ADHD Rating Scales) and PSQI (Pittsburgh Sleep Quality Index). The IQ of the healthy control group and volunteers with ADHD diagnosis were measured using MWT (Multiple-Choice Word Test). The chronotypes of the participants were determined by the D-MEQ (Morning-Eveningness Questionnaire, German Version). No special cognitive testing was implemented in the study. Comorbidities were observed: $28.6 \%$ of participants with ADHD diagnosis has additionally adipositas, $7.1 \%$ has additionally addiction disorder, and $21.4 \%$ has additionally affective disorder. The remaining participants with ADHD diagnosis had no comorbidities.

The four manuscripts of this special issue dealing with circadian rhythmicity describe unique research 
questions (Faltraco et al. 2021a, b, c). Although some samples have been used for more than one research question, the overall sample composition differs from each other and thus is different for each study. Experiments differ substantially in their conditions, thus, they each investigate unique cellular biochemical pathways.

\section{Actigraphy}

To obtain objective measures of participants' sleep and circadian rhythm function, the rest-activity pattern of participants was recorded using wrist-worn actigraphs (Actiwatch 2, Philips Respironics, USA). Actigraphs were worn on the non-dominant wrist for a period of at least 7 consecutive days. The recording interval of the device was set at 60 -s epochs. Data occurring before the first and after the final midnight of each record were excluded, ensuring at least 6 complete days for each participant, with a complete weekend included in each record.

\section{Tissue isolation and fibroblast cell culture}

Human dermal fibroblasts (HDF) were isolated and cultured as described previously (Takashima 1998). Fibroblasts were cultivated $\left(37^{\circ} \mathrm{C}, 5 \% \mathrm{CO}_{2}\right)$ in Dulbecco's Modified Eagle Medium DMEM (Gibco, Thermo Fisher, UK)/1 mg/ml Liberase $^{\mathrm{TM}}$ (Roche, Germany) containing 100 units/ml penicillin, $100 \mu \mathrm{g} / \mathrm{ml}$ streptomycin (Gibco, Thermo Fisher, UK) and $10 \%$ fetal bovine serum FBS (Gibco, Thermo Fisher, UK).

\section{Measurement of cell viability}

Upon confluency of the respective primary fibroblast cell culture from each participant, cells were incubated with $0 \mu \mathrm{M}, 0.1 \mu \mathrm{M}$ and $1.0 \mu \mathrm{M}$ norepinephrine (Arterenol, SanofiAventis, Germany). Following 24 h, cell viability was measured using the Trypan Blue Exclusion Test (Strober 2015).

\section{Measurement of circadian gene expression}

Upon confluency of the respective primary fibroblast cell culture from each participant, eight-culture-flask replicates were prepared and cells were incubated with either $0.1 \mu \mathrm{M}$ or 1.0 $\mu \mathrm{M}$ norepinephrine (NE, Arterenol, Sanofi-Aventis, Germany). Cultures without NE were used as a negative control. After $24 \mathrm{~h}$ of incubation, the cells were synchronized with $100 \mathrm{nM}$ dexamethasone (Sigma-Aldrich, Germany) for $30 \mathrm{~min}$. Samples were harvested every fourth hour after synchronization for a period of $28 \mathrm{~h}$ in solution $\mathrm{D}$ (4.5 M guanidinium thiocyanate, $0.5 \%$ sodium- $N$-lauryl sarcosine, $25 \mathrm{mM}$ tri-sodium citrate, $0.1 \mathrm{M}$ betamercaptoethanol) and stored at $-70{ }^{\circ} \mathrm{C}$. Total RNA was isolated and purified with RNeasy Plus Mini Kit (Qiagen, Germany) as well as subjected to reverse transcription using the Superscript III First-Strand Synthesis System (Invitrogen, Germany). Gene expression of Clock, Bmall, Perl, Per2, Per3 and Cryl as well as housekeeping genes (Rpl13A, Rpl19A, $G A P D H)$ was measured by real-time quantitative reverse transcriptase polymerase chain reaction (qRT-PCR) with CFX Connect ${ }^{\mathrm{TM}}$ Real-Time PCR Detection System (Biorad, Germany). The oligonucleotide sequences are presented in Table 1. All primers were purchased from Eurofins (Alameda, CA). The qRT-PCR was performed in 96-well 0.1-ml thin-wall PCR plates (Applied Biosystems) in the CFX Connect $^{\mathrm{TM}}$ Real-Time PCR Detection System (Biorad, München, Germany). Each $20 \mu \mathrm{l}$ reaction contained $10 \mu \mathrm{l} \mathrm{Kappa} \mathrm{SYBR}$ Green Master Mix (Kappa Biosystems, Darmstadt, Germany), $200 \mathrm{nM}$ gene-specific forward and reverse primer mix (Eurofins, Alameda, CA) and 20 ng template DNA. The expression levels of genes of interest were normalized to the geometrical mean of expression level of housekeeping genes Rpl13A, Rpl19A, and GAPDH from the same sample (Mane et al. 2008). The primer efficiency (between 1.89 and 2.00) was evaluated using the RegLinePCR v 11.0 (Heart Failure Research Center). Data were analyzed using the $\Delta \Delta \mathrm{Ct}$ method (Livak and Schmittgen 2001). The values were normalized to corresponding individual averages.
Table 1 Oligonucleotides for qRT-PCR to measure circadian gene expression

\begin{tabular}{lll}
\hline Gene & Forward primer $\left(5^{\prime}-3^{\prime}\right)$ & Reverse primer $\left(5^{\prime}-3^{\prime}\right)$ \\
\hline Clock & CCAGCAGTTTCATGAGATGC & GAGGTCATTTCATAGCTGAGC \\
Bmal1 & AAGGATGGCTGTTCAGCACATGA & CAAAAATCCATCTGCTGCCCTG \\
Per 1 & TGGGGACAACAGAACAGAGAA & AGGACACTCCTGCGACCA \\
Per 2 & GTATCCATTCATGCTGGGCT & TCGTTTGAACTGCGGTGAC \\
Per3 & TCAGTGTTTGGTGGAAGGAA & TCTGGGTCAGCAGCTCTACA \\
Cry1 & CACGAATCACAAACAGACGG & TACATCCTGGACCCCTGG \\
$R P L 13 a$ & GCCAGAAATGTTGATGCCTT & AGATGGCGGAGGTGCAG \\
$R P L 19 a$ & GTGGCAAGAAGAAGGTCTGG & GCCCATCTTTGATGAGCTTC \\
GAPDH & GAAGGTGAAGGTCGGAGT & GAAGATGGTGATGGGATTTC \\
\hline
\end{tabular}




\section{Statistical methods}

Circadian gene expression data were tested for significant circadian rhythmicity, using CircWave v. 1.4 software (generated by Dr. Roelof Hut; http://www.euclock.org) to determine the best-fitting linear harmonic regression with an assumed period of $24 \mathrm{~h}$ and with $\alpha$ set at 0.05 . The center of gravity of each best-fitting waveform in CircWave was used as the circadian acrophase, and the associated estimation error was used as the SD. Inferential statistics were carried out in SPSS (IBM Corporation).

Actigraphic data were analyzed via MANCOVAs, with age, sex and in some cases ADHD symptom severity included in the model as covariates.

qRT-PCR clock gene data were analyzed via ANOVA and mixed between-within ANOVAs. For all inferential tests, $p<0.05$ was used to indicate a statistically significant groupwise difference. Sample sizes were calculated via GPower 3.1 software; for correlations, the assumptions used were significance level of $\alpha=0.05$ and the power of 0.8 for two groups (ADHD, HC) with three measures $(0 \mu \mathrm{M}, 0.1 \mu \mathrm{M}$ and $1 \mu \mathrm{M} \mathrm{NE}$ ). Although research in this field is generally scarce, we assumed that the influence of $\mathrm{NE}$ on the circadian gene expression will have an effect size $d^{\prime}=0.5$, returning a required total sample size of 21 . Taking into consideration an expected drop-out rate, $n=12$ participants were allocated to each group. One-way ANOVA was used to assess differences of clock gene expression levels among chronotype groups. Associations between clock gene expression and chronotype obtained from the healthy controls and ADHD participants were studied by Spearman's rank order correlation. Data were analyzed via time series statistics adequately powered by 12 samples each, which in this statistical model is mathematically sufficient and thus representative (Menet et al. 2012; Thaben and Westermark 2016).

\section{Results}

\section{Demographic data}

Human dermal fibroblasts (HDF) were obtained via skin biopsy from healthy controls (HC) (4 men, 8 women; $41.50 \pm 14.04$ years, mean \pm SD; BMI: $25.87 \pm 5.42 \mathrm{~kg} /$ $\mathrm{m}^{2}$, mean $\pm \mathrm{SD}$ ) and volunteers with diagnosed ADHD (9 men, 5 women; $41.57 \pm 13.45$ years, mean \pm SD; BMI: $26.21 \pm 3.62 \mathrm{~kg} / \mathrm{m}^{2}$, mean $\pm \mathrm{SD}$ ). All participants completed the Multiple-Choice Word Test (IQ score: HC: $110.25 \pm 9.32$, mean \pm SD; ADHD participants: $108.86 \pm 12.60$, mean \pm SD, n.s), Morningness-Eveningness Questionnaire, German Version (D-MEQ score: HC: $58.83 \pm 8.97$, mean \pm SD; ADHD participants: $46.57 \pm 15.44$, mean $\pm \mathrm{SD}, p=0.024$ ) and Wender Utah Rating Scale, German Short Version
(WURS-k score: HC: $7.17 \pm 8.19$, mean \pm SD; ADHD participants: $37.21 \pm 15.20$, mean $\pm \mathrm{SD}, p<0.001)$. The demographic data are presented in Table 2 .

There were no significant differences in age, BMI, IQ or gender across the two study groups. D-MEQ scores indicated that ADHD patients displayed more definitive and moderate evening preference than healthy controls.

\section{Actigraphy}

Measures from the non-parametric circadian rhythm analysis were analyzed across the two groups, healthy controls and ADHD participants, in a MANCOVA with age and sex as co-variates. For two participants, actigraphy analysis was not completed. No statistical significant effect of group was observed (Pillai's trace $=0.205 ; F=0.643 ; p=0.695$; partial ETA squared $=0.205$ ). Bonferroni post hoc correction showed no significant difference for mid-sleep on weekend days ( $p=0.774)$, mid-sleep on weekdays $(p=0.382)$, social jetlag $(p=0.553)$, sleep efficiency $(p=0.975)$, WASO (wakening after sleep onset; $p=0.927$ ) and total number of wake bouts $(p=0.659)$.

Measures from the non-parametric circadian rhythm analysis were analyzed across the two groups, healthy controls and ADHD participants, in a MANCOVA with chronotype as co-variate. No statistical significant effect of group was observed (Pillai's trace $=0.116 ; F=0.351 ; p=0.899$; partial ETA squared $=0.116$ ). A significant difference for mid-sleep on weekend days $(p=0.008)$, mid-sleep on weekdays $(p=0.001)$, but not for social jetlag $(p=0.928)$, sleep efficiency ( $p=0.715$ ), WASO (wakening after sleep onset; $p=0.925)$ and total number of wake bouts $(p=0.570)$ was observed (Fig. 1).

\section{Cell viability}

The viability of the cultivated human dermal fibroblasts (HDF) after norepinephrine (NE) incubation was compared with HDFs without NE. The viability of cells treated with NE $(0.1 \mu \mathrm{M}$ NE: $94.01 \pm 1.73$, mean \pm SD; $1.0 \mu \mathrm{M}$ NE: $93.23 \pm 2.19$, mean $\pm \mathrm{SD}$ ) was marginally

Table 2 Demographic data

\begin{tabular}{lll}
\hline Demographic data & Healthy controls, $n=12$ & ADHD, $n=14$ \\
\hline Age & $41.50 \pm 14.04$ years & $41.57 \pm 13.45$ years \\
Female & $8(66.7 \%)$ & $5(35.4 \%)$ \\
BMI & $25.87 \pm 5.42$ & $26.21 \pm 3.62$ \\
IQ score & $110.25 \pm 9.32$ & $108.86 \pm 12.60$ \\
D-MEQ & $58.83 \pm 8.97 *$ & $46.57 \pm 15.44 *$ \\
WURS-k-score & $7.17 \pm 8.19^{* * *}$ & $37.21 \pm 15.20^{* * *}$ \\
\hline
\end{tabular}

$* p<0.05, * * * p<0.001$ 

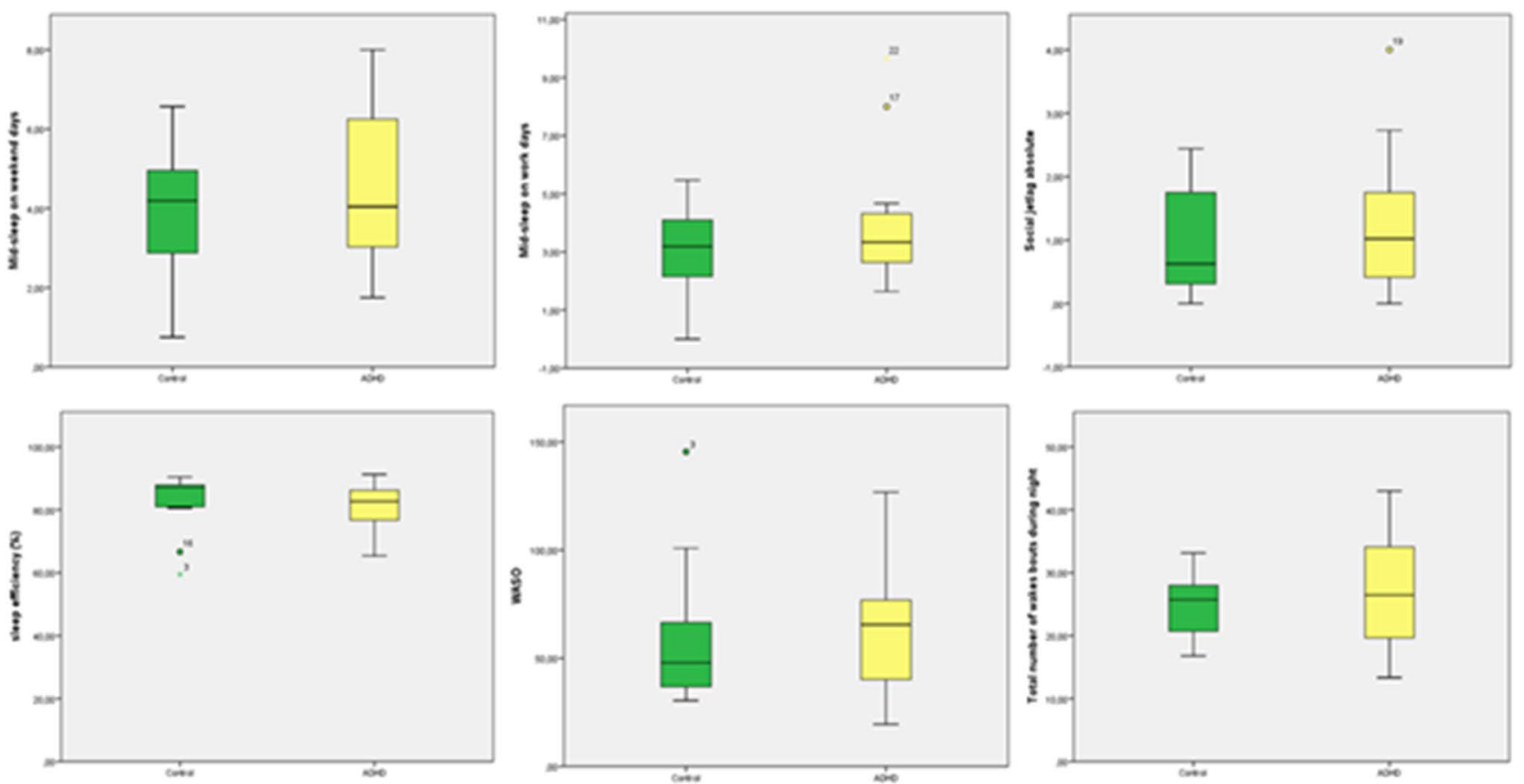

Fig. 1 Actigraphic measures of mid-sleep of weekend days, mid-sleep of week days, social jetlag, sleep efficiency, WASO (wakening after sleep onset) and total number of wake bouts are displayed as boxplots. Circles correspond to outlier values and asterisks correspond to extreme values

decreased compared to control cells without NE $(0 \mu \mathrm{M}$ NE: $95.44 \pm 1.19$, mean \pm SD).

\section{Circadian gene expression in human dermal fibroblasts}

The expression profiles of six circadian genes after incubation with different NE concentrations were examined in primary fibroblasts cultured from skin biopsies and synchronized with dexamethasone. Bmall, Cryl, Per $2 /$ Per 3 expression was strongly rhythmic in both groups (CircWave, $p<0.01)$. No rhythmicity was detected for Clock in both groups except for HC group with $0.1 \mu \mathrm{M} \mathrm{NE}$ (CircWave, $p=0.015)$. In the ADHD group, $1 \mu \mathrm{M}$ NE exposure dampened the rhythmicity of Perl (CircWave, $p>0.05$ ).

Gene expression in healthy participants revealed a statistical significant difference between cultures incubated with $\mathrm{NE}$ and negative controls (without NE incubation), as determined by one-way ANOVA for Bmall at ZT12 $(F=5.043$, $p=0.012)$, Perl at ZT20 $(F=4.730, p=0.016)$ and Per3 at ZT4 $(F=5.594, p=0.008)$ and ZT28 $(F=3.674, p=0.037)$. A Bonferroni post hoc analysis revealed a significant lower expression in cultures incubated with $1 \mu \mathrm{M} \mathrm{NE}$ compared to negative controls for Bmall (ZT12, $p=0.029$ ), Perl (ZT20, $p=0.018$ ) and Per3 (ZT28, $p=0.045)$. The Bmall expression at ZT12 $(p=0.029)$ was significant lower among the cultures incubated with $0.1 \mu \mathrm{M}$ and $1 \mu \mathrm{M}$ NE. The Per 3 expression was significant higher at ZT4 $(p=0.007)$ between cultures incubated with $1 \mu \mathrm{M}$ NE and negative controls. One-way ANOVA in the ADHD group revealed no statistical significant differences between cultures incubated with $\mathrm{NE}$ and negative controls (Supplement Fig. S1).

Mixed between-within ANOVA analysis of circadian gene data with group as between-subjects factor and time as within-subject factor showed significant main effects for time for all circadian genes $(p<0.01)$. Per3 expression did show a significant $\mathrm{ZT} \times$ group interaction via mixed ANOVA (Greenhouse-Geisser corrected $F_{2.58,11.87}=1.856, p=0.046$, partial ETA squared $=0.140$ ). Bonferroni post hoc correction showed Per3 expression at ZT4 to be significant higher in the healthy controls incubated with $1 \mu \mathrm{M} \mathrm{NE}$ compared to the participants of the $\mathrm{HC}$ group without NE incubation $(p=0.050)$ as well as between healthy controls incubated with $1 \mu \mathrm{M}$ NE and ADHD cultures without NE incubation $(p=0.030)$. The Per 3 expression differed between controls and ADHD group at ZT28 ( $p=0.012)$ (Fig. 2).

Although the Bmall expression was strongly rhythmic in both groups, there was no $\mathrm{ZT} \times$ group interaction via mixed ANOVA (Greenhouse-Geisser corrected $F_{0.36,26.40}=1.346$, $p=0.122$, partial ETA squared $=0.091$ ). No ZT $\times$ group interaction via mixed ANOVA was observed for Clock (Greenhouse-Geisser corrected $F_{0.19,25.88}=0.866, p=0.656$, partial ETA squared $=0.064)$, Cryl $($ Greenhouse-Geisser corrected $F_{0.26,24.53}=1.084, p=0.360$, partial ETA squared $=0.075)$, Perl (Greenhouse-Geisser corrected $F_{0.73,27.34}=0.872, p=0.654$, partial ETA squared $=0.063$ ) 


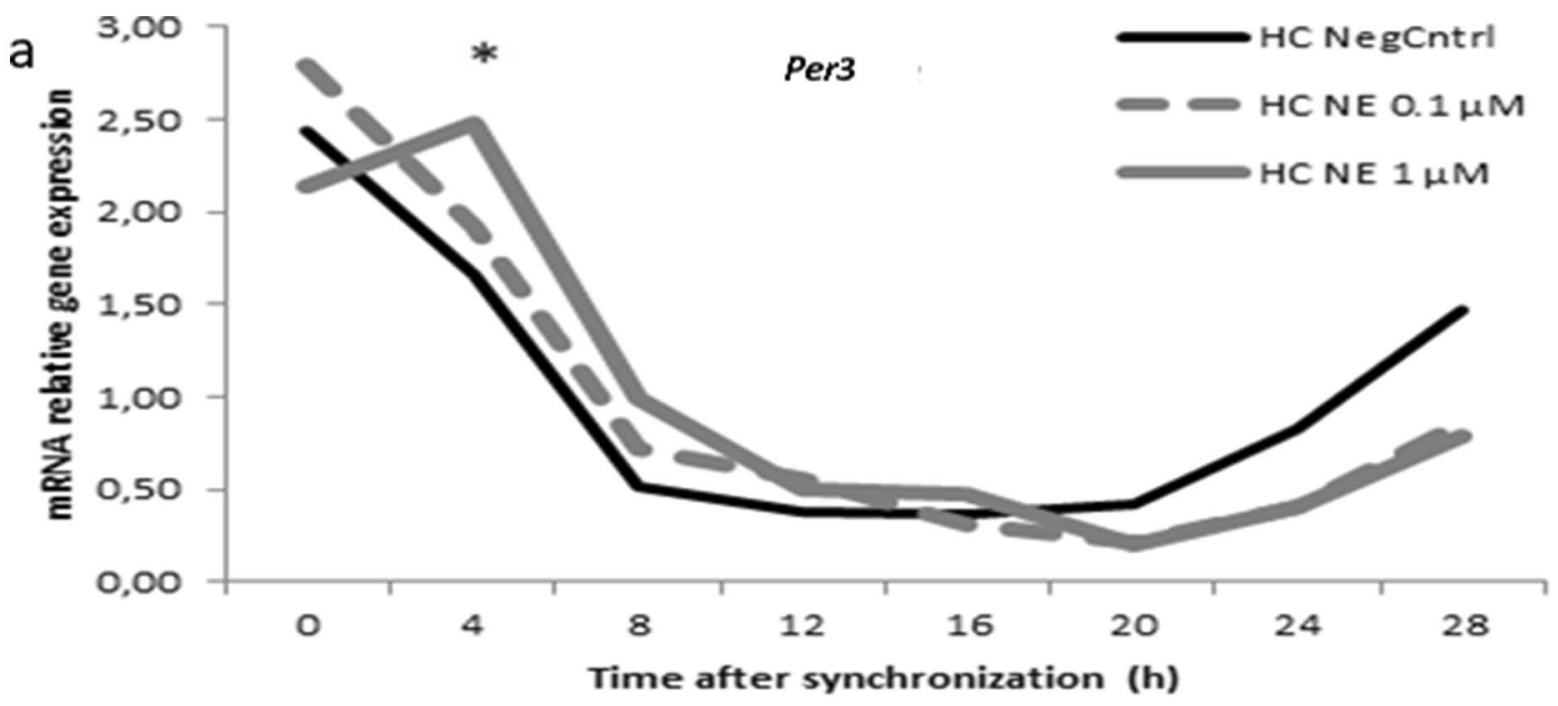

b
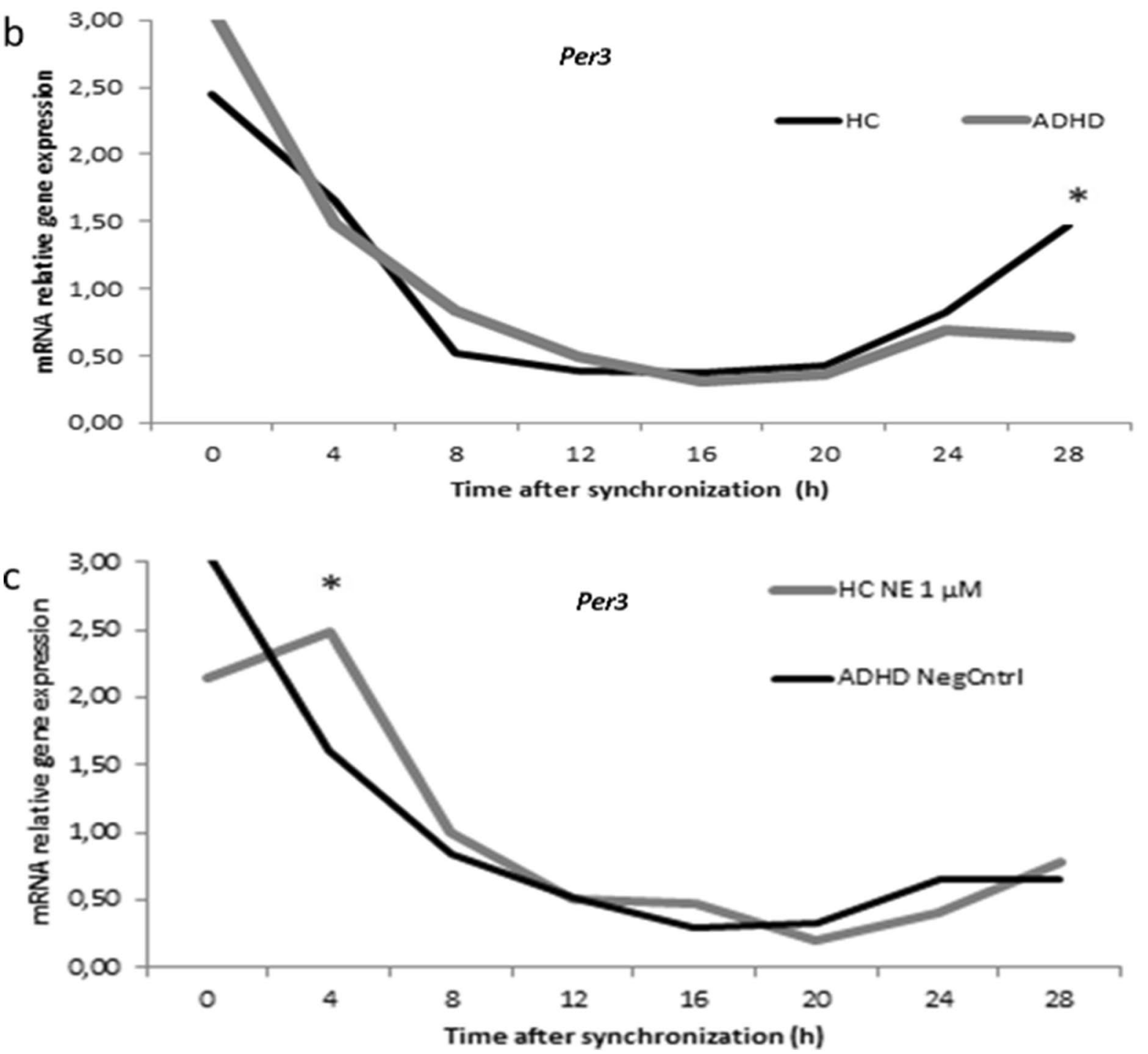
4Fig. 2 a Relative mRNA gene expression of Per3 in healthy controls $(0,0.1,1 \mu \mathrm{M} \mathrm{NE})$. b Relative mRNA gene expression of Per3 between healthy controls and ADHD volunteers for $0 \mu \mathrm{M}$ NE. c Relative mRNA gene expression of Per3 between healthy controls $(1 \mu \mathrm{M})$ and ADHD volunteers for $(0 \mu \mathrm{M} \mathrm{NE})$. Asterisks correspond to significant values

and Per2 (Greenhouse-Geisser corrected $F_{0.87,18.18}=0.874$, $p=0.269$, partial ETA squared $=0.086$ ) expression.

Clock, Bmall and Cryl resulted in a slight amplitude and phase differences between HC group and ADHD group after NE treatment, whereas, Perl, Per2 and Per3 after NE incubation lead to an adjustment to the expression of these Per genes to the control group (Fig. 3).

\section{Chronotype and circadian gene expression}

$58.3 \%$ of healthy participants displayed a neutral preference, whereas $25.0 \%$ presented moderate morning and $16.7 \%$ definite morning preference. Among the ADHD group 35.7\% participants displayed moderate morning and $28.6 \%$ neutral preference. In the ADHD group, the evening preference was represented by $21.4 \%$ definite evening and $14.3 \%$ moderate evening type. The chronotype group data are presented in Table 3.

Differences of clock gene expression levels among chronotype were assessed using one-way ANOVA. Gene expression in healthy participants revealed a statistical significant difference between chronotypes for Bmall at ZT16 $(F=4.884, p=0.037)$ and Per3 at ZT0 $(F=6.802$, $p=0.016)$. A Bonferroni post hoc correction revealed a significant lower expression of Bmall $(p=0.040)$ and Per3 $(p=0.018)$ in healthy participants exhibiting neutral type compared to those with moderate morning type. In ADHD participants, Bmall expression presented differences at ZT8 $(F=6.630, p=0.013)$, particularly between moderate morning and definite evening type (Bonferroni post hoc test, $p=0.013$ ). In HC cultures incubated with NE, one-way ANOVA revealed a statistical significant gene expression differences between chronotypes for Perl (with $0.1 \mu \mathrm{M} \mathrm{NE}$, $F=6.680, p=0.019$ ) and Per2 at ZT0 (with $0.1 \mu \mathrm{M} \mathrm{NE}$, $F=6.802, p=0.016$ ), as well as Clock (with $1.0 \mu \mathrm{M} \mathrm{NE}$, $F=4.639, p=0.041)$ at ZT24. Bonferroni post hoc correction showed significant differences of Perl expression levels at ZT16 $(p=0.018)$ between neutral type and moderate morning chronotypes. When compared to definite morning chronotype, the expression of Per2 at ZT16 $(p=0.031)$ and Clock at ZT24 ( $p=0.050)$ was significant higher in healthy participants with neutral chronotype.

In ADHD cultures incubated with NE, several significant differences were observed. One-way ANOVA revealed a statistical significant differences in gene expression after $0.1 \mu \mathrm{M}$ NE between chronotypes for Clock $(F=7.654$, $p=0.006)$, Cryl $(F=7.365, p=0.007), \operatorname{Perl}(F=5.747$, $p=0.017)$ and $\operatorname{Per} 2(F=9.109, p=0.003)$. Bonferroni post hoc analysis showed differences in expression between evening chronotypes. The participants with definite evening preference had a higher Cryl at ZT0 $(p=0.014)$ and Perl at ZT4 $(p=0.022)$, as well as a lower Perl at ZT24 $(p=0.039)$ compared to ADHD participants with moderate evening preference. In participants with moderate evening preference, the expression of Perl at ZT24 was significant higher compared to those exhibiting neutral $(p=0.005)$ and moderate morning preference $(p=0.012)$. Same differences were observed for Per2 at ZT0 $(p=0.003)$ between the moderate evening and moderate morning chronotypes. Participants with an ADHD diagnosis with moderate morning chronotype revealed a higher Clock expression at ZT4 $(p=0.005)$ compared to moderate evening chronotype and a lower Cryl expression at ZT0 ( $p=0.003)$ compared to definite evening chronotype. After $1 \mu \mathrm{M}$ NE, Perl expression presented differences at ZT28 $(F=5.592, p=0.023)$, particularly between moderate morning and moderate evening chronotypes (Bonferroni post hoc test, $p=0.024$ ).

A Spearman's rank order correlation showed the relationship between chronotype and clock gene relative expressions. There was a strong positive correlation for chronotype and Bmall (ZT16, $r_{s}=0.611, p=0.035$ ), Cryl (ZT16, $\left.r_{s}=0.591, p=0.043\right)$ and Per3 gene (ZT0, $r_{s}=0.631$, $p=0.028$ ) in HC cultures. In HC cultures incubated with $\mathrm{NE}$, a strong positive correlation for chronotype with Bmall (HC with $0.1 \mu \mathrm{M} \mathrm{NE}$ at ZT28, $r_{s}=0.611, p=0.035$; HC with $1 \mu \mathrm{M}$ NE at ZT16, $\left.r_{s}=0.670, p=0.017\right)$ and Per3 gene (HC with $0.1 \mu \mathrm{M} \mathrm{NE}$ at ZT0, $r_{s}=0.710, p=0.010$ ) was observed. In the ADHD group, chronotype and Bmall gene expressions were positively correlated, at ZT4 $\left(r_{s}=0.714\right.$, $p=0.004)$ and ZT8 $\left(r_{s}=0.892, p<0.0001\right)$, as well as Cryl (ZT8, $\left.r_{s}=0.544, p=0.044\right)$ and Clock at ZT12 $\left(r_{s}=0.620\right.$, $p=0.018)$. In the ADHD cultures incubated with NE, a positive correlation between chronotype and Clock (ADHD with $0.1 \mu \mathrm{M} \mathrm{NE}$ at ZT4, $r_{s}=0.574, p=0.031$ ), Cryl (ADHD with $0.1 \mu \mathrm{M} \mathrm{NE}$ at ZT4, $r_{s}=0.547, p=0.043$ ), Perl (ADHD with $1 \mu \mathrm{M} \mathrm{NE}$ at ZT20, $r_{s}=0.554, p=0.040$ ) and Per2 (ADHD with $1 \mu \mathrm{M} \mathrm{NE}$ at ZT24, $\left.r_{s}=0.628, p=0.039\right)$ was observed.

\section{Discussion}

The results of the present study illustrate that ADHD is associated to alterations in the circadian rhythm. It demonstrates that norepinephrine (NE) impacts significantly on circadian function. The expression and rhythm of all Per genes in the ADHD group adjusted to the healthy control (HC) group after NE incubation.

Previous studies have indicated that circadian processes are altered in neuropsychiatric disorders such as ADHD 

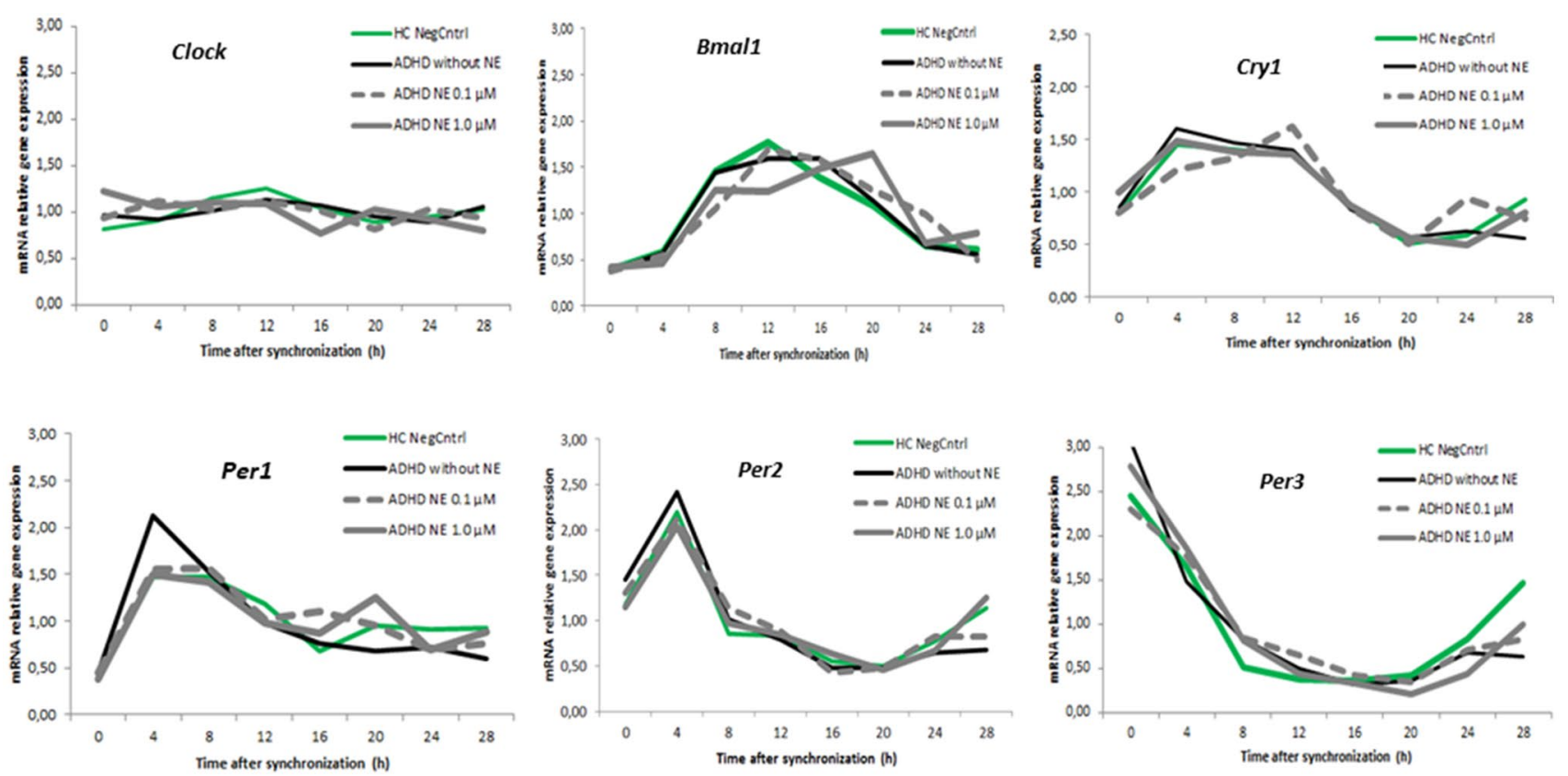

Fig. 3 Relative mRNA gene expression of circadian genes in healthy controls $(0 \mu \mathrm{M})$ and ADHD volunteers $(0,0.1,1 \mu \mathrm{M} N \mathrm{~N})$

Table 3 Chronotype groups and demographic data in healthy and ADHD participants

\begin{tabular}{|c|c|c|c|c|c|c|c|}
\hline \multirow{2}{*}{$\begin{array}{l}\text { Demographic data } \\
\text { in healthy controls }\end{array}$} & \multirow[t]{2}{*}{ All $(n=12)$} & \multicolumn{2}{|l|}{ Morning type } & \multirow[t]{2}{*}{ Neutraltype $(n=7)$} & \multicolumn{2}{|l|}{ Evening type } & \multirow{2}{*}{$\begin{array}{l}p \text { value } \\
\text { (one-way } \\
\text { ANOVA) }\end{array}$} \\
\hline & & $\begin{array}{l}\text { Moderate morn- } \\
\text { ing type }(n=3)\end{array}$ & $\begin{array}{l}\text { Definite } \\
\text { morning type } \\
(n=2)\end{array}$ & & $\begin{array}{l}\text { Moderate even- } \\
\text { ing type }\end{array}$ & $\begin{array}{l}\text { Definite even- } \\
\text { ing type }\end{array}$ & \\
\hline Age (years) & $41.50 \pm 14.04$ & $43.00 \pm 15.72$ & $56.00 \pm 7.68$ & $36.57 \pm 12.83$ & N/A & N/A & ns \\
\hline Gender & 4 male, 8 female & 3 female & 2 female & 4 male, 3 female & N/A & N/A & ns \\
\hline BMI & $25.86 \pm 5.42$ & $25.06 \pm 4.05$ & $25.55 \pm 0.63$ & $26.30 \pm 6.91$ & N/A & N/A & ns \\
\hline D-MEQ & $58.83 \pm 8.97$ & $65.33 \pm 2.88$ & $72.00 \pm 1.41$ & $52.29 \pm 3.95$ & N/A & N/A & $9.10 \mathrm{E}-05$ \\
\hline WURS-k & $7.17 \pm 8.18$ & $6.67 \pm 7.02$ & $1.00 \pm 0.00$ & $9.14 \pm 9.44$ & N/A & N/A & ns \\
\hline IQ & $110.25 \pm 9.32$ & $106.67 \pm 7.50$ & $99.50 \pm 6.36$ & $114.86 \pm 8.00$ & N/A & N/A & ns \\
\hline \multirow{2}{*}{$\begin{array}{l}\text { Demographic data } \\
\text { in ADHD }\end{array}$} & \multirow[t]{2}{*}{ All $(n=14)$} & \multicolumn{2}{|l|}{ Morning } & \multirow[t]{2}{*}{ Neutraltype $(n=4)$} & \multicolumn{2}{|l|}{ Evening type } & \multirow{2}{*}{$\begin{array}{l}p \text { value } \\
\text { (one-way } \\
\text { ANOVA) }\end{array}$} \\
\hline & & $\begin{array}{l}\text { Moderate morn- } \\
\text { ing type }(n=5)\end{array}$ & $\begin{array}{l}\text { Definite morn- } \\
\text { ing type }\end{array}$ & & $\begin{array}{l}\text { Moderate even- } \\
\text { ing type }(n=2)\end{array}$ & $\begin{array}{l}\text { Definite } \\
\text { evening type } \\
(n=3)\end{array}$ & \\
\hline Age (years) & $41.57 \pm 13.45$ & $42.20 \pm 15.55$ & N/A & $45.50 \pm 11.62$ & $47.00 \pm 16.97$ & $31.67 \pm 11.72$ & ns \\
\hline Gender & 9 male, 5 female & 3 male, 2 female & N/A & 2 male, 2 female & 1 male, 1 female & 3 male & ns \\
\hline BMI & $26.21 \pm 3.64$ & $26.15 \pm 3.76$ & N/A & $27.05 \pm 4.78$ & $26.40 \pm 4.10$ & $25.06 \pm 3.42$ & ns \\
\hline D-MEQ & $46.57 \pm 15.44$ & $63.00 \pm 3.08$ & N/A & $47.50 \pm 3.31$ & $36.50 \pm 2.12$ & $24.67 \pm 3.78$ & $1.14 \mathrm{E}-07$ \\
\hline WURS-k & $37.21 \pm 15.20$ & $41.00 \pm 13.57$ & N/A & $32.75 \pm 10.63$ & $17.00 \pm 7.07$ & $50.33 \pm 13.86$ & ns \\
\hline IQ & $108.86 \pm 12.60$ & $106.80 \pm 7.25$ & N/A & $109.25 \pm 19.61$ & $120.00 \pm 2.82$ & $104.33 \pm 13.43$ & ns \\
\hline
\end{tabular}

(Baird et al. 2012; Coogan et al. 2016, 2019; Coogan and McGowan 2017; Cronin et al. 2017; Faltraco et al. 2020; Johansson et al. 2016; Korman et al. 2018; Lippert et al. 2014; Mansour et al. 2017; McCarthy et al. 2013a; Yang et al. 2009). Our group reported that patients with ADHD using ADHD-medication (methylphenidate and atomoxetine) have altered sleep activity compared to both controls and ADHD participants without medication. At the molecular level, there were alterations in the expression of Per2 and Cryl between ADHD individuals with no medication compared to medicated ADHD patients or HC, whereas Clock expression was altered in patients with ADHD using 
ADHD-medication (Coogan et al. 2019). The observed medication effect in the ADHD group is attributed to norepinephrine modulation. Second-line pharmacological treatment for ADHD besides stimulant methylphenidate, is atomoxetine (ATO), a selective NET inhibitor which increases $\mathrm{NE}$ levels. Extensive loss of NE terminals has been linked to alterations in brain regions which are vital for cognition, mood, and executive function (Phillips et al. 2016) as well as alterations of the circadian clock (Andrade-Silva et al. 2014; Li and Cassone 2015; Morioka et al. 2010; Terazono et al. 2003).

We observed several changes in the rhythmic expression of Per3 gene. Cultures from ADHD patients with no NE resulted in lower expression of Per $328 \mathrm{~h}$ after dexamethasone synchronization than either controls or cultures with NE. Expression of Per 3 was higher in the controls with $1 \mu \mathrm{M}$ NE cultures at ZT4 compared to cultures without NE.

Animal studies demonstrated that alterations of $\mathrm{mBmall}$, mPer 1 and $m$ Per 2 as well as BBmall, gClock, gCryl and gCry 2 are associated with NE pathways ( $\mathrm{Li}$ and Cassone 2015; Terazono et al. 2003), however, no alteration of Per3 linked to NE has been reported until now.

Chronotype is an important influence on circadian clock gene expression (Pegoraro et al. 2015; Takahashi et al. 2018). In Drosophila melanogaster, gene expression was associated with early and late chronotypes (Pegoraro et al. 2015). Takahashi and colleagues observed that chronotype and social jetlag were related to the rhythm of clock gene expression in 24 young adults with a significant main effect of time for Per3 and nuclear receptor subfamily NR1D1 and NR2D2 in the morningness group (Andrade-Silva et al. 2014; Li and Cassone 2015; Morioka et al. 2010; Takahashi et al. 2018; Terazono et al. 2003).

A variable number tandem repeat (VNTR) polymorphism in Per3 (Dijk and Archer 2010) has been associated with chronotype, sleep homeostasis and various psychiatric disorders (Andrade-Silva et al. 2014; Li and Cassone 2015; Morioka et al. 2010; Terazono et al. 2003). No association between the Per3 VNTR and diurnal preference was observed among Norwegian university students (AndradeSilva et al. 2014; Li and Cassone 2015; Morioka et al. 2010; Terazono et al. 2003). Another study including one hundred and eighty eight healthy young adults, observed no effect for chronotype and circadian gene polymorphism of Clock and Per3 (McGowan et al. 2020).

We observed changes in the circadian gene expression levels among healthy and ADHD participants with different chronotypes. After exposure of HDF cultures to NE, more significant differences were observed, particularly between ADHD participants exhibiting morning and evening preferences. Our results show a strong significant correlation of chronotype with gene expression $16 \mathrm{~h}$ after dexamethasone synchronization for Bmall and Cryl genes, and immediately after synchronization for Per3. After incubation of HDF cultures obtained from healthy controls with NE, the positive correlation of Bmall was shifted to $28 \mathrm{~h}$ after cell synchronization. In ADHD participants, the chronotype is positively correlated with Clock, Bmall and $C r y l$ genes in the first $12 \mathrm{~h}$ after synchronization. In the ADHD group, the exposure to NE reveals a positive correlation of chronotype with Clock and Cryl at ZT4, as well as with Per1/2 at ZT20 and ZT24.

Some studies have suggested that chronotype is associated with weight gain that may induce changes in the circadian gene expressions. A downregulation of Per1, Per2, Per3, Nrld2 and PAR-domain basic leucine zipper transcription factors $D b p$, Tef was observed in adipose tissues of mice after weight fluctuations (Dankel et al. 2014). A prospective study of weight gain associated with chronotype among college freshmen reports that individuals with eveningness chronotype have a significantly greater BMI compared with morningness and neutral types (Culnan et al. 2013). In our study, the healthy individuals with neutral chronotype have a slightly higher BMI $(26.30 \pm 6.91)$ compared to the morningness type $(25.31 \pm 2.34)$. This applies also to the ADHD participants, with neutral type having a higher BMI $(27.05 \pm 4.78)$ than both the morningness chronotype $(26.15 \pm 3.76)$ and the eveningness chronotype $(25.73 \pm 3.76)$.

A study by Lucassen et al. determined that adults who present an eveningness preference and sleep less than $6.5 \mathrm{~h}$ a day have more $24 \mathrm{~h}$ urinary epinephrine levels (Lucassen et al. 2013). In addition, the plasma and urinary NE are elevated in patients with obstructive sleep apnea syndrome (Fletcher 2003). A study reported circadian gene dysfunction in patients with obstructive sleep apnea syndrome, particularly changes in Perl mRNA expression (Burioka et al. 2008). In the same study, it was observed that administration of NE induced Perl mRNA in the cerebral cortex of mice in vivo (Burioka et al. 2008).

In summary, NE influences the circadian clock in human dermalfibroblasts from study participants with a diagnosis of ADHD.

It is to mention, that no special cognitive testing was implemented in this study. In addition, the participants of the ADHD group took no medication before and during the study. For further studies, a connection between circadian disturbances, cognitive deficits and the effect of medication would be suitable.

Supplementary Information The online version contains supplementary material available at https://doi.org/10.1007/s00702-021-02376-2.

Acknowledgements 2020 FORUN: This project has received funding from the University Medicine Rostock under Grant Agreement No. 889024. 2020 CoCA ADHD Comorbidity. This project has received 
funding from the European Union's Horizon 2020 research and innovation programme under Grant Agreement No. 667302.

Funding Open Access funding enabled and organized by Projekt DEAL. 2020 FORUN: Thisproject has received funding from the University Medicine Rostock under Grant Agreement No. 889024. 2020 CoCA ADHD Comorbidity. This project has received funding from the European Union's Horizon 2020 research and innovation programme under Grant Agreement No. 667302.

Data availability Data and material are available.

Code availability Actiwatch 2, Philips Respironics, USA; CircWave v. 1.4 software (generated by Dr. Roelof Hut; http://www.euclock.org); SPSS (IBM Corporation).

\section{Declarations}

Conflict of interest Johannes Thome has received financial support from pharmaceutical companies (Actelion, Astra Zeneca, BristolMyers Squibb, EVER Neuro Pharma GmbH, Janssen-Cilag, Lilly, Lundbeck, MEDICE, Merz, Novartis, Pfizer, Roche, Servier, Shire, Trommsdorff) some of which manufacture medication used in the treatment of ADHD patients. Frank Faltraco, Oliver Tucha, Frederick Simon, Adriana Uzoni and Denise Palm have no potential conflict of interest to disclose.

Ethical approval Ethical approval for the conduct of the study, including obtaining human dermal biopsy samples, was given by the ethical review committee of Rostock University (Registration-number: A2013-159).

Consent to participate Written consent was obtained from each study participant.

Consent for publication Written consent was obtained from each study participant.

Open Access This article is licensed under a Creative Commons Attribution 4.0 International License, which permits use, sharing, adaptation, distribution and reproduction in any medium or format, as long as you give appropriate credit to the original author(s) and the source, provide a link to the Creative Commons licence, and indicate if changes were made. The images or other third party material in this article are included in the article's Creative Commons licence, unless indicated otherwise in a credit line to the material. If material is not included in the article's Creative Commons licence and your intended use is not permitted by statutory regulation or exceeds the permitted use, you will need to obtain permission directly from the copyright holder. To view a copy of this licence, visit http://creativecommons.org/licenses/by/4.0/.

\section{References}

Andrade-Silva J, Cipolla-Neto J, Peliciari-Garcia RA (2014) The in vitro maintenance of clock genes expression within the rat pineal gland under standard and norepinephrine-synchronized stimulation. Neurosci Res 81-82:1-10. https://doi.org/10.1016/j. neures.2014.03.005

Baird AL, Coogan AN, Siddiqui A, Donev RM, Thome J (2012) Adult attention-deficit hyperactivity disorder is associated with alterations in circadian rhythms at the behavioural, endocrine and molecular levels. Mol Psychiatry 17:988-995. https://doi.org/10. 1038/mp.2011.149

Beane M, Marrocco RT (2004) Norepinephrine and acetylcholine mediation of the components of reflexive attention: implications for attention deficit disorders. Prog Neurobiol 74:167-181. https:// doi.org/10.1016/j.pneurobio.2004.09.001

Biederman J, Spencer T (1999) Attention-deficit/hyperactivity disorder (ADHD) as a noradrenergic disorder. Biol Psychiatry 46:1234-1242

Burioka N et al (2008) Clock gene dysfunction in patients with obstructive sleep apnoea syndrome. Eur Respir J 32:105-112. https://doi. org/10.1183/09031936.00138207

Coogan AN, McGowan NM (2017) A systematic review of circadian function, chronotype and chronotherapy in attention deficit hyperactivity disorder. Atten Deficit Hyperact Disord 9:129-147. https://doi.org/10.1007/s12402-016-0214-5

Coogan AN, Baird AL, Popa-Wagner A, Thome J (2016) Circadian rhythms and attention deficit hyperactivity disorder: the what, the when and the why. Prog Neuropsychopharmacol Biol Psychiatry 67:74-81. https://doi.org/10.1016/j.pnpbp.2016.01.006

Coogan AN et al (2019) Impact of adult attention deficit hyperactivity disorder and medication status on sleep/wake behavior and molecular circadian rhythms. Neuropsychopharmacology 44:1198-1206. https://doi.org/10.1038/s41386-019-0327-6

Cronin P et al (2017) Circadian alterations during early stages of Alzheimer's disease are associated with aberrant cycles of DNA methylation in BMAL1. Alzheimers Dement 13:689-700. https:// doi.org/10.1016/j.jalz.2016.10.003

Culnan E, Kloss JD, Grandner M (2013) A prospective study of weight gain associated with chronotype among college freshmen. Chronobiol Int 30:682-690. https://doi.org/10.3109/07420 528.2013.782311

Czeisler CA et al (1999) Stability, precision, and near-24-hour period of the human circadian pacemaker. Science 284:2177-2181

Dankel SN et al (2014) Weight cycling promotes fat gain and altered clock gene expression in adipose tissue in C57BL/6 J mice. Am J Physiol Endocrinol Metab 306:E210-224. https://doi.org/10.1152/ ajpendo.00188.2013

Dijk DJ, Archer SN (2010) PERIOD3, circadian phenotypes, and sleep homeostasis Sleep Med Rev 14:151-160. https://doi.org/ 10.1016/j.smrv.2009.07.002

Durgan DJ et al (2005) The intrinsic circadian clock within the cardiomyocyte. Am J Physiol Heart Circ Physiol 289:H1530-1541. https://doi.org/10.1152/ajpheart.00406.2005

Faltraco F, Uzoni A, Shevchuk L, Thome J, Palm D (2020) Synchronization of fibroblasts ex vivo in psychopharmacology. Pharmacopsychiatry. https://doi.org/10.1055/a-1151-4947

Faltraco F, Palm D, Uzoni A, Simon F, Tucha O, Thome J (2021a) Atomoxetine and circadian gene expression in human dermal fibroblasts from participants with a diagnosis of attention-deficit hyperactivity disorder. J Neural Transm. https://doi.org/10.1007/ s00702-021-02373-5 (Accepted)

Faltraco F, Palm D, Uzoni A, Borchert L, Simon F, Tucha O, Thome J (2021b) Dopamine adjusts the circadian gene expression of Per2 and Per3 in human dermal fibroblasts from ADHD patients. J Neural Transm. https://doi.org/10.1007/s00702-021-02374-4 (Accepted)

Faltraco F, Palm D, Coogan A, Uzoni A, Duwe I, Simon F, Tucha O, Thome J (2021c) Remdesivir shifts circadian rhythmicity to eveningness; similar to the most prevalent chronotype in ADHD. J Neural Transm. https://doi.org/10.1007/s00702-021-02375-3 (Accepted)

Fletcher EC (2003) Sympathetic over activity in the etiology of hypertension of obstructive sleep apnea. Sleep 26:15-19. https://doi. org/10.1093/sleep/26.1.15 
Gillbro JM, Marles LK, Hibberts NA, Schallreuter KU (2004) Autocrine catecholamine biosynthesis and the beta-adrenoceptor signal promote pigmentation in human epidermal melanocytes. J Investig Dermatol 123:346-353. https://doi.org/10.1111/j.0022-202X. 2004.23210.x

Johansson AS, Owe-Larsson B, Hetta J, Lundkvist GB (2016) Altered circadian clock gene expression in patients with schizophrenia. Schizophr Res 174:17-23. https://doi.org/10.1016/j.schres.2016. 04.029

Korman M, Palm D, Uzoni A, Faltraco F, Tucha O, Thome J, Coogan AN (2018) ADHD 24/7: circadian clock genes, chronotherapy and sleep/wake cycle insufficiencies in ADHD. World J Biol Psychiatry. https://doi.org/10.1080/15622975.2018.1523565

Li Y, Cassone VM (2015) Clock-controlled regulation of the acute effects of norepinephrine on chick pineal melatonin rhythms. J Biol Rhythms 30:519-532. https://doi.org/10.1177/0748730415 607060

Lippert J et al (2014) Altered dynamics in the circadian oscillation of clock genes in dermal fibroblasts of patients suffering from idiopathic hypersomnia. PLoS ONE 9:e85255. https://doi.org/10. 1371/journal.pone.0085255

Livak KJ, Schmittgen TD (2001) Analysis of relative gene expression data using real-time quantitative PCR and the 2(-Delta Delta C(T)) method. Methods 25:402-408. https://doi.org/10.1006/ meth.2001.1262

Lucassen EA et al (2013) Evening chronotype is associated with changes in eating behavior, more sleep apnea, and increased stress hormones in short sleeping obese individuals. PLoS ONE 8:e56519. https://doi.org/10.1371/journal.pone.0056519

MacDonald E, Kobilka BK, Scheinin M (1997) Gene targeting-homing in on alpha 2-adrenoceptor-subtype function. Trends Pharmacol Sci 18:211-219

Mane VP, Heuer MA, Hillyer P, Navarro MB, Rabin RL (2008) Systematic method for determining an ideal housekeeping gene for real-time PCR analysis. J Biomol Tech 19:342-347

Mansour HA et al (2017) Associations between period 3 gene polymorphisms and sleep- /chronotype-related variables in patients with late-life insomnia. Chronobiol Int 34:624-631. https://doi.org/10. 1080/07420528.2017.1287083

Marien MR, Colpaert FC, Rosenquist AC (2004) Noradrenergic mechanisms in neurodegenerative diseases: a theory. Brain Res Brain Res Rev 45:38-78. https://doi.org/10.1016/j.brainresrev.2004.02. 002

McCarthy MJ, Fernandes M, Kranzler HR, Covault JM, Welsh DK (2013a) Circadian clock period inversely correlates with illness severity in cells from patients with alcohol use disorders. Alcohol Clin Exp Res 37:1304-1310. https://doi.org/10.1111/acer.12106

McCarthy MJ, Wei H, Marnoy Z, Darvish RM, McPhie DL, Cohen BM, Welsh DK (2013b) Genetic and clinical factors predict lithium's effects on PER2 gene expression rhythms in cells from bipolar disorder patients. Transl Psychiatry 3:e318. https://doi. org/10.1038/tp.2013.90

McGowan NM, Uzoni A, Faltraco F, Thome J, Coogan AN (2020) The impact of social jetlag and chronotype on attention, inhibition and decision making in healthy adults. J Sleep Res. https://doi.org/10. $1111 /$ jsr. 12974

Menet JS, Rodriguez J, Abruzzi KC, Rosbash M (2012) Nascent-Seq reveals novel features of mouse circadian transcriptional regulation. Elife 1:e00011. https://doi.org/10.7554/eLife.00011

Moore RY (1997) Circadian rhythms: basic neurobiology and clinical applications. Annu Rev Med 48:253-266. https://doi.org/10.1146/ annurev.med.48.1.253

Morioka N, Sugimoto T, Tokuhara M, Dohi T, Nakata Y (2010) Noradrenaline induces clock gene Per1 mRNA expression in C6 glioma cells through $\beta 2$-adrenergic receptor coupled with protein kinase A - cAMP response element binding protein (PKACREB) and Src-tyrosine kinase - glycogen synthase kinase-3 $\beta$ (Src-GSK-3 $\beta$ ). J Pharmacol Sci 113:234-245. https://doi.org/10. 1254/jphs.10031FP

Pegoraro M, Picot E, Hansen CN, Kyriacou CP, Rosato E, Tauber E (2015) Gene expression associated with early and late chronotypes in Drosophila melanogaster. Front Neurol 6:100. https://doi.org/ 10.3389/fneur.2015.00100

Phillips C, Fahimi A, Das D, Mojabi FS, Ponnusamy R, Salehi A (2016) Noradrenergic system in down syndrome and Alzheimer's disease a target for therapy. Curr Alzheimer Res 13:68-83. https:// doi.org/10.2174/1567205012666150921095924

Reppert SW, Weaver DR (2002) Coordination of circadian timing in mammals. Nature 418:935-941

Sharma A, Couture J (2014) A review of the pathophysiology, etiology, and treatment of attention-deficit hyperactivity disorder (ADHD). Ann Pharmacother 48:209-225. https://doi.org/10.1177/ 1060028013510699

Simonneaux V, Ribelayga C (2003) Generation of the melatonin endocrine message in mammals: a review of the complex regulation of melatonin synthesis by norepinephrine, peptides, and other pineal transmitters. Pharmacol Rev 55:325-395. https://doi.org/10.1124/ pr.55.2.2

Steinkraus V, Mak JC, Pichlmeier U, Mensing H, Ring J, Barnes PJ (1996) Autoradiographic mapping of beta-adrenoceptors in human skin. Arch Dermatol Res 288:549-553

Strober W (2015) Trypan blue exclusion test of cell viability. Curr Protoc Immunol 111:A3 B 1-A3 B 3. https://doi.org/10.1002/04711 42735.ima03bs 111

Takahashi M et al (2018) Chronotype and social jetlag influence human circadian clock gene expression. Sci Rep 8:10152. https://doi.org/ 10.1038/s41598-018-28616-2

Takashima A (1998) Establishment of fibroblast cultures. Curr Protoc Cell Biol Chapter 2:Unit 2.1

Terazono H, Mutoh T, Yamaguchi S, Kobayashi M, Akiyama M, Udo R, Ohdo S, Okamura H, Shibata S (2003) Adrenergic regulation of clock gene expression in mouse liver. Proc Natl Acad Sci USA 100:6795

Terbeck S, Savulescu J, Chesterman LP, Cowen PJ (2016) Noradrenaline effects on social behaviour, intergroup relations, and moral decisions. Neurosci Biobehav Rev 66:54-60. https://doi.org/10. 1016/j.neubiorev.2016.03.031

Thaben PF, Westermark PO (2016) Differential rhythmicity: detecting altered rhythmicity in biological data. Bioinformatics 32:28002808. https://doi.org/10.1093/bioinformatics/btw309

Xing B, Li YC, Gao WJ (2016) Norepinephrine versus dopamine and their interaction in modulating synaptic function in the prefrontal cortex. Brain Res 1641:217-233. https://doi.org/10.1016/j.brain res.2016.01.005

Xu FYSH, Liu AC, Takahashi JS, Kay SA (2000) Mice lacking the norepinephrine transporter are supersensitive to psychostimulants. Nat Neurosci 3:465-471

Yang S, Van Dongen HP, Wang K, Berrettini W, Bucan M (2009) Assessment of circadian function in fibroblasts of patients with bipolar disorder. Mol Psychiatry 14:143-155. https://doi.org/10. 1038/mp.2008.10

Publisher's Note Springer Nature remains neutral with regard to jurisdictional claims in published maps and institutional affiliations. 